\title{
Positive Solutions for a Class of Discrete Mixed Boundary Value Problems with the $(p, q)$-Laplacian Operator
}

\author{
Cuiping $\mathrm{Li}^{1}$ and Zhan Zhou $\mathbb{D}^{1,2}$ \\ ${ }^{1}$ School of Mathematics and Information Science, Guangzhou University, Guangzhou 510006, China \\ ${ }^{2}$ Center for Applied Mathematics, Guangzhou University, Guangzhou 510006, China \\ Correspondence should be addressed to Zhan Zhou; zzhou0321@hotmail.com
}

Received 31 December 2019; Accepted 15 February 2020; Published 26 March 2020

Academic Editor: Beatrice Di Bella

Copyright ( $) 2020$ Cuiping Li and Zhan Zhou. This is an open access article distributed under the Creative Commons Attribution License, which permits unrestricted use, distribution, and reproduction in any medium, provided the original work is properly cited.

In this paper, we consider the existence of solutions for the discrete mixed boundary value problems involving $(p, q)$-Laplacian operator. By using critical points theory, we obtain the existence of at least two positive solutions for the boundary value problem under appropriate assumptions on the nonlinearity.

\section{Introduction}

In recent years, with the development of mechanical engineering, control system, computer science, and economics, the existence of solutions of difference equations has attracted wide attention (see [1-6]). For example, applying Ricceri variational principle to obtain the existence of multiple solutions [7-9], taking the invariant sets of descending flow to prove the existence of sign-changing solutions [10], making the linking theorem to get the existence and multiplicity of periodic solutions [11], and using critical point theory to obtain the existence of homoclinic solutions [12-15] and heteroclinic solutions [16].

As we know, the fixed-point method and upper and lower solution techniques are important tools to solve the existence of solutions for boundary value problems (see
$[17,18])$. But recently, it is more common to use critical point theory to study Dirichlet boundary value problems (see [19-23]). More result on difference equations by using critical point theories can be referred to [24-27].

In [28], D'Aguì et al. established the existence of at least two positive solutions for the following discrete Dirichlet boundary value problem:

$$
\left\{\begin{array}{l}
-\Delta\left(\phi_{p}(\Delta u(k-1))\right)+q(k) \phi_{p}(u(k))=\lambda f(k, u(k)), \quad k \in \mathbb{Z}(1, N), \\
u(0)=u(N+1)=0,
\end{array}\right.
$$

where $q(k) \geq 0$ for all $k \in\{1,2, \ldots, N\}$.

Unlike this, D'Aguì et al. in [29] proved that there are at least two nonzero weak solutions for the following mixed boundary value problem:

$$
\left\{\begin{array}{l}
-\left(q(x)\left|u^{\prime}(k)\right|^{p-2} u^{\prime}(x)\right)^{\prime}+s(x)|u(k)|^{p-2} u(x)=\lambda f(x, u(x)), \quad x \in[a, b] \\
u(a)=u^{\prime}(b)=0
\end{array}\right.
$$

where $p>1, q, s \in L^{\infty}([a, b])$ with $q_{0}=\operatorname{essinf}_{[a, b]} q>0, s_{0}=$

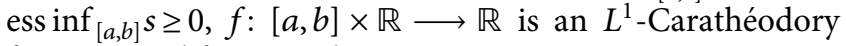
function and $\lambda$ is a real positive parameter.
As a discrete analogy of the abovementioned problem, we consider the existence of positive solutions for the following discrete mixed boundary value problem: 


$$
\left\{\begin{array}{l}
-\Delta\left(\phi_{p}(\Delta u(k-1))\right)+s(k) \phi_{q}(u(k))=\lambda f(k, u(k)), k \in \mathbb{Z}(1, N), \quad\left(D_{\lambda}^{f}\right) \\
u(0)=\Delta u(N)=0,
\end{array}\right.
$$

where $\mathbb{Z}(a, b)$ denote the discrete interval $\{a, a+1, \ldots, b\}$ for any integers $a$ and $b$ with $a<b, N$ be a positive integer, $f(k, u)$ is continuous in $u$ for each $k \in \mathbb{Z}(1, N), \Delta u(k)=$ $u(k+1)-u(k)$ is the forward difference operator, $\phi_{r}: \mathbb{R} \longrightarrow \mathbb{R}$ is the $r$-Laplacian given by $\phi_{r}(u)=|u|^{r-2} u$ with $u \in \mathbb{R}, 1<q \leq p<+\infty, s(k) \geq 0$ for all $k \in \mathbb{Z}(1, N)$, and $\lambda$ is a positive parameter.

In this paper, under suitable assumptions on the nonlinearity $f$, we use the theory of two nonzero critical points (see [30]) to ensure that there are at least two nonzero solutions for problem $\left(D_{\lambda}^{f}\right)$. The two nonzero critical points theorem is an appropriate combination of local minimum theorem (see [31]) and classical Ambrosetti-Rabinowitz theorem (see [32]). An important hypothesis of mountain pass theorem is Palais-Smale condition. It satisfies the application of infinite dimensional space by requiring the condition that the nonlinear term is stronger than $p$ superlinearity at infinity. In order to obtain the existence of two nonzero solutions, we can assume the classical Ambrosetti-Rabinowitz condition and nonlinear algebraic condition (see (40) in Theorem 2) hold, that is, more widespread than the $p$-sublinearity at zero. Moreover, when we require that $f(k, 0) \geq 0$ for all $k \in \mathbb{Z}(1, N)$, we can use strong maximum principle to obtain the existence of positive solutions, which has been proved in Lemma 2 .

Let $s_{*}=\min \{s(k): k \in \mathbb{Z}(1, N)\}$, a special case of our main result is stated as follows.

Theorem 1. Let $f: \mathbb{R} \longrightarrow \mathbb{R}$ be a continuous function such that

$$
\begin{array}{cc}
\lim _{t \rightarrow 0^{+}} \frac{f(t)}{t^{p-1}} & 4(\mathrm{a}) \quad \text { Proposition 1. The following inequality holds: } \\
\lim _{t \longrightarrow+\infty} \frac{f(t)}{t^{p-1}} & 4(\mathrm{~b}) \\
\|u\|_{\infty} \leq \max \left\{\left(\frac{p N^{p-1}}{1+s_{*} N^{p-1}}\right)^{1 / q}\left(\frac{\|u\|^{p}}{p}+\frac{\sum_{k=1}^{N} s(k)|u(k)|^{q}}{q}\right)^{1 / q},\left(\frac{p N^{p-1}}{1+s_{*} N N^{-1}}\right)^{1 / p}\left(\frac{\|u\|^{p}}{p}+\frac{\sum_{k=1}^{N} s(k)|u(k)|^{q}}{q}\right)^{1 / p}\right\} .
\end{array}
$$

then, for each $\lambda \in\left(0,\left(1+s_{*} N^{p-1} / p N^{p}\right) \min \left\{s u p_{c>0}\left(c^{q}\right)\right.\right.$ $\left.\left.\left.\max _{|\xi| \leq c} \int_{0}^{\xi} f(t) d t\right), \sup _{c>0}\left(c^{p / \max _{|\xi| \leq c}} \int_{0}^{\xi} f(t) d t\right)\right\}\right)$, the problem

$\left\{\begin{array}{l}-\Delta\left(\phi_{p}(\Delta u(k-1))\right)+s(k) \phi_{q}(u(k))=\lambda f(u(k)), \quad k \in \mathbb{Z}(1, N), \\ u(0)=\Delta u(N)=0\end{array}\right.$

admits at least two positive solutions.

The structure of the article is as follows. In Section 2, some basic definitions and properties are given. In Section 3, we give the main results. Under suitable hypothesis, Lemma 1 is used to obtain that the problem $\left(D_{\lambda}^{f}\right)$ possesses at least two positive solutions. Finally, some examples are given to illustrate our main results.

In this section, we recall some definitions, notations, and properties. Consider the $N$-dimensional Banach space:

$$
S=\{u: \mathbb{Z}(0, N+1) \longrightarrow \mathbb{R}: u(0)=\Delta u(N)=0\},
$$

and define the norm

$$
\|u\|=\left(\sum_{k=1}^{N+1}|\Delta u(k-1)|^{p}\right)^{1 / p}
$$

and $\|u\|_{\infty}=\max \{|u(k)|: k \in \mathbb{Z}(1, N)\}$ is another norm in $S$.

\section{Preliminaries}

Proof. Let $u \in S$, then there exist $k^{*} \in \mathbb{Z}(1, N)$ such that

Since $\left|u\left(k^{*}\right)\right|=\max \{|u(k)|: k \in \mathbb{Z}(1, N)\}$.

$$
\left|u\left(k^{*}\right)\right|=\left|\sum_{k=1}^{k^{*}} \Delta u(k-1)\right| \leq\left(\sum_{k=1}^{k^{*}} 1\right)^{1-1 / p}\left(\sum_{k=1}^{k^{*}}|\Delta u(k-1)|^{p}\right)^{1 / p} \leq N^{1-1 / p}\|u\|
$$


then

$$
\begin{aligned}
& \|u\|_{\infty}^{p} \leq N^{p-1}\|u\|^{p} . \quad \text { If }\|u\|_{\infty}>1 \text {, then } \\
& \frac{\left(1+s_{*} N^{p-1}\right)\|u\|_{\infty}^{q}}{p} \leq \frac{\|u\|_{\infty}^{p}+N^{p-1} \sum_{k=1}^{N} s(k)|u(k)|^{q}}{p} \\
& \leq \frac{N^{p-1}}{p}\left(\|u\|^{p}+\sum_{k=1}^{N} s(k)|u(k)|^{q}\right) \leq \frac{N^{p-1}}{p}\|u\|^{p}+\frac{N^{p-1}}{q} \sum_{k=1}^{N} s(k)|u(k)|^{q},
\end{aligned}
$$

that is,

$$
\|u\|_{\infty} \leq\left(\frac{p N^{p-1}}{1+s_{*} N^{p-1}}\right)^{1 / q}\left(\frac{\|u\|^{p}}{p}+\frac{\sum_{k=1}^{N} s(k)|u(k)|^{q}}{q}\right)^{1 / q} .
$$

If $\|u\|_{\infty} \leq 1$, then

$$
\begin{aligned}
\frac{\left(1+s_{*} N^{p-1}\right) u_{\infty}^{p}}{p} & \leq \frac{\|u\|_{\infty}^{p}+N^{p-1} \sum_{k=1}^{N} s(k)|u(k)|^{q}}{p} \\
& \leq \frac{N^{p-1}}{p}\|u\|^{p}+\frac{N^{p-1}}{q} \sum_{k=1}^{N} s(k)|u(k)|^{q},
\end{aligned}
$$

that is,

$$
\|u\|_{\infty} \leq\left(\frac{p N^{p-1}}{1+s_{*} N^{p-1}}\right)^{1 / p}\left(\frac{\|u\|^{p}}{p}+\frac{\sum_{k=1}^{N} s(k)|u(k)|^{q}}{q}\right)^{1 / p} .
$$

In summary, we have

$$
\begin{aligned}
& \begin{array}{l}
\|u\|_{\infty} \leq \max \left\{( \frac { p N ^ { p - 1 } } { 1 + s _ { * } N ^ { p - 1 } } ) ^ { 1 / q } \left(\frac{\|u\|^{p}}{p}+\frac{\sum^{2}}{\text { Put }}\right.\right. \\
F(k, t):=\int_{0}^{t} f(k, \xi) \mathrm{d} \xi, \quad \forall(k, t) \in \mathbb{Z}(1, N) \\
\text { consider the function } J_{\lambda}: S \longrightarrow \mathbb{R} \text { for al } \\
\qquad J_{\lambda}=\Phi-\lambda \Psi, \\
\qquad \Phi:=\Phi_{1}+\Phi_{2}, \Phi_{1}(u)=\frac{\|u\|^{p}}{p},
\end{array} \\
& \Phi_{2}(u)=\frac{\sum_{k=1}^{N} s(k)|u(k)|^{q}}{q}, \\
& \Psi(u):=\sum_{k=1}^{N} F(k, u(k)) .
\end{aligned}
$$

where

It is clear that $\Phi_{1}, \Phi_{2}, \Psi \in C^{1}(S, \mathbb{R})$ and their Gâteaux derivatives at the point $u \in S$ are given by

$$
\Phi_{1}^{\prime}(u)(v)=\sum_{k=1}^{N+1} \phi_{p}(\Delta u(k-1)) v(k)
$$$$
=-\sum_{k=1}^{N+1} \phi_{p}(\Delta u(k-1)) v(k-1)
$$$$
=\sum_{k=1}^{N} \phi_{p}(\Delta u(k-1)) v(k)-\sum_{k=0}^{N} \phi_{p}(\Delta u(k)) v(k)
$$$$
=-\sum_{k=1}^{N} \Delta \phi_{p}(\Delta u(k-1)) v(k),
$$$$
\Phi_{2}^{\prime}(u)(v)=\sum_{k=1}^{N} s(k) \phi_{q}(u(k)) v(k)
$$$$
\Psi^{\prime}(u)(v)=\sum_{k=1}^{N} f(k, u(k)) v(k),
$$ 
for all $u, v \in S$. So, we have

$$
J_{\lambda}^{\prime}(u)(v)=\sum_{k=1}^{N}\left[-\Delta\left(\phi_{p}(\Delta u(k-1))\right)+s(k) \phi_{q}(u(k))-\lambda f(k, u(k))\right] v(k) .
$$
$\left(D_{\lambda}^{f}\right)$.

Hence, a critical point $u$ of $J_{\lambda}$ is a solution of problem

Now, we recall a definition and a two nonzero critical points theorem for the reader's convenience.

Definition 1. Let $X$ be a real Banach space; we say that a Gâteaux differentiable function $J_{\lambda}: X \longrightarrow \mathbb{R}$ satisfies the (PS)-condition, if any sequence $\left\{u_{n}\right\}_{n \in N} \subseteq X$ such that

(i) $J_{\lambda}\left(u_{n}\right) \longrightarrow c \in \mathbb{R}$, as $n \longrightarrow+\infty$

(ii) $J_{\lambda}^{\prime}\left(u_{n}\right) \longrightarrow 0$, as $n \longrightarrow+\infty$, has a convergent subsequence

Lemma 1. Let $X$ be a real Banach space and $\Phi, \Psi \in C^{1}(S, \mathbb{R})$ such that $\inf _{X}(\Phi)=\Phi(0)=\Psi(0)=0$. Assume that there are $r \in \mathbb{R}$ and $\omega \in X$, with $0<\Phi(\omega)<r$, such that

$$
\frac{\sup _{u \in \Phi^{-1}(-\infty, r]} \Psi(u)}{r}<\frac{\Psi(\omega)}{\Phi(\omega)},
$$

and for each

$$
\lambda \in \Lambda=\left(\frac{\Phi(\omega)}{\Psi(\omega)}, \frac{r}{\sup _{u \in \Phi^{-1}(-\infty, r]} \Psi(u)}\right),
$$

the functional $J_{\lambda}=\Phi-\lambda \Psi$ satisfies the (PS)-condition and it is unbounded from below.

Then, for each $\lambda \in \Lambda$, the functional $J_{\lambda}$ admits at least two nonzero critical points $u_{\lambda, 1}, u_{\lambda, 2}$ such that $J_{\lambda}\left(u_{\lambda, 1}\right)<0$ $<J_{\lambda}\left(u_{\lambda, 2}\right)$.

In order to obtain the positive solution of problem $\left(D_{\lambda}^{f}\right)$, we establish the following strong maximum principle.

Lemma 2. Fix $u \in S$ such that either

$$
u(k)>0 \text { or }-\Delta\left(\phi_{p}(\Delta u(k-1))\right)+s(k) \phi_{q}(u(k)) \geq 0,
$$

for all $k \in \mathbb{Z}(1, N)$. Then, either $u>0$ in $\mathbb{Z}(1, N)$ or $u \equiv 0$.

Proof. Let $j \in \mathbb{Z}(1, N)$ such that

$$
u(j)=\min \{u(k): k \in \mathbb{Z}(1, N)\} .
$$

If $u(j)>0$, then it is easy know that $u>0$ in $\mathbb{Z}(1, N)$. If $u(j) \leq 0$, then by $(23)$, we have

$$
-\Delta\left(\phi_{p}(\Delta u(j-1))\right) \geq-s(j) \phi_{q}(u(j)) \geq 0,
$$

that is,

$$
\phi_{p}(\Delta u(j)) \leq \phi_{p}(\Delta u(j-1)) .
$$

Since $\phi_{p}(u)$ is increasing in $u$, we have

$$
\Delta u(j) \leq \Delta u(j-1) \text {. }
$$

By the definition of $u(j)$, we know that

$$
\begin{aligned}
\Delta u(j) & \geq 0, \\
\Delta u(j-1) & \leq 0 .
\end{aligned}
$$

By combining (27) with (28), we get $u(j+1)=u(j)=$ $u(j-1)$. If $j-1=0$, we have $u(j)=u(j-1)=0$. Otherwise, $j-1 \in \mathbb{Z}(1, N)$, replacing $j-1$ by $j$, we know $u(j-2)=u(j-1)$. Continuing in this way, we have $u(j)=u(j-1)=\cdots=u(0)=0$. Similarly, we have $u(j)=u(j+1)=\cdots=u(N+1)$. Thus, $u(k)=u(0)=0$ and $\forall k \in \mathbb{Z}(1, N)$.

Now, put

$$
F^{+}(k, t)=\int_{0}^{t} f\left(k, \xi^{+}\right) \mathrm{d} \xi, \quad \forall(k, t) \in \mathbb{Z}(1, N) \times \mathbb{R},
$$

where $\xi^{+}=\max \{\xi, 0\}$

Define $\quad J_{\lambda}^{+}=\Phi_{1}+\Phi_{2}-\lambda \Psi^{+} \quad$ and $\Psi^{+}(u):=\sum_{k=1}^{N} F^{+}(k, u(k))$. Standard arguments show that $J_{\lambda}^{+} \in C^{1}(S, R)$ and the critical points of $J_{\lambda}^{+}$are precisely the solutions of the following problem:

$$
\left\{\begin{array}{l}
-\Delta\left(\phi_{p}(\Delta u(k-1))\right)+s(k) \phi_{q}(u(k))=\lambda f\left(k, u^{+}(k)\right), \quad k \in \mathbb{Z}(1, N), \quad\left(D_{\lambda}^{f^{+}}\right) \\
u(0)=\Delta u(N)=0 .
\end{array}\right.
$$

Lemma 3. If $f(k, 0) \geq 0$ for each $k \in \mathbb{Z}(1, N)$, any nonzero critical point of the functional $J_{\lambda}^{+}$is a positive solution of problem $\left(D_{\lambda}^{f}\right)$.
Proof. Since a critical point of $J_{\lambda}^{+}$is a solution of problem $D_{\lambda}^{f^{+}}$, the conclusion follows by the discrete maximum principle ([33], Proposition 1). 
Next, we suppose that $f(k, 0) \geq 0$ and $f(k, x)=f(k, 0)$ for all $x \leq 0$ and for all $k \in \mathbb{Z}(1, N)$. Put

$$
\begin{aligned}
L_{\infty} & :=\min _{k \in \mathbb{Z}(1, N)} \liminf _{t \longrightarrow+\infty} \frac{F(k, t)}{t^{p}}, \\
\widetilde{s} & =\sum_{k=1}^{N} s(k),
\end{aligned}
$$

we have the following result.
Proof. Let $\lambda>2^{p} N+\widetilde{s}-2^{p-1} / q L_{\infty}$. We consider a sequence $\left\{u_{n}\right\}_{n \in N} \subseteq S$ such that $J_{\lambda}\left(u_{n}\right) \longrightarrow c \in \mathbb{R}$ and $J_{\lambda}^{\prime}\left(u_{n}\right) \longrightarrow 0$, as $n \longrightarrow+\infty$. Let $u_{n}^{+}=\max \left\{u_{n}, 0\right\}$ and $u_{n}^{-}=\max \left\{-u_{n}, 0\right\}$ for all $n \in N$. We first prove that $\left\{u_{n}^{-}\right\}$is bounded. On one hand, we have

$$
\begin{gathered}
\left|\Delta u_{n}^{-}(k-1)\right|^{p} \leq-\phi_{p}\left(\Delta u_{n}(k-1)\right) \Delta u_{n}^{-}(k-1), \\
s(k)\left|u_{n}^{-}(k)\right|^{q}=-s(k)\left|u_{n}(k)\right|^{q-2} u_{n}(k) u_{n}^{-}(k),
\end{gathered}
$$

for all $k \in \mathbb{Z}(1, N)$. So,

Lemma 4. If $L_{\infty}>0$, then $J_{\lambda}$ satisfies $(P S)$-condition and it unbounded from below for all $\lambda \in\left(2^{p} N+\widetilde{s}-2^{p-1} /\right.$ $\left.q L_{\infty},+\infty\right)$.

$$
\begin{aligned}
\left\|u_{n}^{-}\right\|^{p} & =\sum_{k=1}^{N+1}\left|\Delta u_{n}^{-}(k-1)\right|^{p} \\
& \leq-\sum_{k=1}^{N+1} \phi_{p}\left(\Delta u_{n}(k-1)\right) u_{n}^{-}(k)+\sum_{k=1}^{N=1} \phi_{p}\left(\Delta u_{n}(k-1)\right) u_{n}^{-}(k-1) \\
& =-\sum_{k=1}^{N} \phi_{p}\left(\Delta u_{n}(k-1)\right) u_{n}^{-}(k)+\sum_{k=1}^{N} \phi_{p}\left(\Delta u_{n}(k)\right) u_{n}^{-}(k) \\
& =\sum_{k=1}^{N} \Delta \phi_{p}\left(\Delta u_{n}(k-1)\right) u_{n}^{-}(k)=-\Phi_{1}^{\prime}\left(u_{n}\right)\left(u_{n}^{-}\right), \\
\sum_{k=1}^{N} s(k)\left|u_{n}^{-}(k)\right|^{q} & =-\sum_{k=1}^{N} s(k) \phi_{q}\left(u_{n}(k)\right) u_{n}^{-}(k)=-\Phi_{1}^{\prime}\left(u_{n}\right)\left(u_{n}^{-}\right) .
\end{aligned}
$$

On the other hand, we assume that

$$
f(k, u)= \begin{cases}f(k, u), & \text { if } u>0, \\ f(k, 0), & \text { if } u \leq 0,\end{cases}
$$

for each $k \in \mathbb{Z}(1, N)$, then

$$
\Psi^{\prime}\left(u_{n}\right)\left(u_{n}^{-}\right)=\sum_{k=1}^{N} f\left(k, u_{n}(k)\right) u_{n}^{-}(k) \geq 0 .
$$

Therefore,

$$
\begin{aligned}
\left\|u_{n}^{-}\right\|^{p} & \leq\left\|u_{n}^{-}\right\|^{p}+\sum_{k=1}^{N} s(k)|u(k)|^{q} \\
& \leq-\Phi_{1}^{\prime}\left(u_{n}\right)\left(u_{n}^{-}\right)-\Phi_{2}^{\prime}\left(u_{n}\right)\left(u_{n}^{-}\right)+\lambda \Psi^{\prime}\left(u_{n}\right)\left(u_{n}^{-}\right) \\
& =-J_{\lambda}^{\prime}\left(u_{n}\right)\left(u_{n}^{-}\right),
\end{aligned}
$$

for all $n \in N$, which leads to $\left\|u_{n}^{-}\right\|^{p-1} \longrightarrow 0$ as $n \longrightarrow+\infty$. So, we have $\left\|u_{n}^{-}\right\| \longrightarrow 0$ as $n \longrightarrow+\infty$. It means that there exists an $M>0$ such that $u_{n}^{-} \leq M$. From (10) we know that $\left\|u_{n}^{-}\right\|_{\infty} \leq N^{1-1 / p} M=\gamma$, for all $k \in \mathbb{Z}(1, N)$.

Next, we suppose that the sequence $\left\{u_{n}\right\}$ is unbounded, that is, $\left\{u_{n}^{+}\right\}$is unbounded.

As $L_{\infty}>0$, we know that there exists an $l \in R$ such that $L_{\infty}>l>2^{p} N+\widetilde{s}-2^{p-1} / \lambda q$. From the definition of $L_{\infty}$, there is $\delta_{k}>0$ such that $F(k, t)>l|t|^{p}$ for all $t>\delta_{k}$. Furthermore, since $F(k, t)$ is a continuous function, there exists a constant $C(k) \geq 0$ such that $F(k, t) \geq l|t|^{p}-C(k)$ with $t \in\left[-\gamma, \delta_{k}\right]$. Thus, $F(k, t) \geq l|t|^{p}-C(k)$ for all $s \geq-\gamma$ and $k \in \mathbb{Z}(1, N)$. We can obtain that

$$
\sum_{k=1}^{N} F\left(k, u_{n}(k)\right) \geq \sum_{k=1}^{N} l\left|u_{n}(k)\right|^{p}-C \geq l\left\|u_{n}\right\|_{\infty}^{p}-C,
$$

for all $k \in \mathbb{Z}(1, N)$, where $C=\sum_{k=1}^{N} C(k)$, that is,

$$
\Psi\left(u_{n}\right) \geq l\left\|u_{n}\right\|_{\infty}^{p}-C .
$$

Hence, for all $u_{n}$ such that $\left\|u_{n}\right\|_{\infty} \geq 1$, we conclude that 


$$
\begin{aligned}
J_{\lambda}\left(u_{n}\right)= & \frac{\sum_{k=1}^{N+1}\left|\Delta u_{n}(k-1)\right|^{p}}{p}+\frac{\sum_{k=1}^{N} s(k)\left|u_{n}(k)\right|^{q}}{q}-\lambda \Psi\left(u_{n}\right) \\
\leq & \frac{2^{p-1}}{p}\left(\sum_{k=1}^{N}\left|u_{n}(k)\right|^{p}+\sum_{k=1}^{N}\left|u_{n}(k-1)\right|^{p}\right) \\
& +\frac{1}{q} \sum_{k=1}^{N} s(k)\left|u_{n}(k)\right|^{q}-\lambda \Psi\left(u_{n}\right) \\
\leq & \frac{2^{p-1}(2 N-1)}{p}\left\|u_{n}\right\|_{\infty}^{p}+\frac{\widetilde{s}_{1}}{q}\left\|u_{n}\right\|_{\infty}^{q}-\lambda \Psi\left(u_{n}\right) \\
\leq & \left(\frac{2^{p} N+\widetilde{s}-2^{p-1}}{q}-\lambda l\right)\left\|u_{n}\right\|_{\infty}^{p}+\lambda C .
\end{aligned}
$$

Since $\quad 2^{p} N+\widetilde{s}-2^{p-1} / q-\lambda l<0$, we can get $\lim _{n \longrightarrow+\infty} J_{\lambda}\left(u_{n}\right)=-\infty$ and this is absurd. Hence, $J_{\lambda}$ satisfies (PS)-condition.
Let $\left\{u_{n}\right\}$ be such that $\left\{u_{n}^{-}\right\}$is bounded and $\left\{u_{n}^{+}\right\}$is unbounded. From the proof above we can see that $J_{\lambda}$ is unbounded from below.

\section{Main Results}

The main results of this paper are as follows.

Theorem 2. Let $f: \mathbb{Z}(1, N) \times \mathbb{R} \longrightarrow \mathbb{R}$ be a continuous function satisfying $f(k, 0) \geq 0$ for all $k \in \mathbb{Z}(1, N)$. If there are two constants $c$ and $d$ with $d<c$ such that

$$
\begin{aligned}
& \frac{p N^{p-1}}{1+s_{*} N^{p-1}} \sum_{k=1}^{N} \max _{|\xi| \leq c} F(k, \xi) \max \left\{\frac{1}{c^{q}}, \frac{1}{c^{p}}\right\} \\
& \quad<\min \left\{\frac{\sum_{k=1}^{N} F(k, d)}{d^{p} p^{-1}+d^{q} q^{-1} \widetilde{s}}, \frac{q L_{\infty}}{2^{p} N+\widetilde{s}-2^{p-1}}\right\} .
\end{aligned}
$$

Then, for each $\lambda \in \Lambda_{1}$ with

$$
\Lambda_{1}:=\left(\max \left\{\frac{d^{p} p^{-1}+d^{q} q^{-1} \widetilde{s}}{\sum_{k=1}^{N} F(k, d)}, \frac{2^{p} N+\widetilde{s}-2^{p-1}}{q L_{\infty}}\right\}, \frac{\left(1+s_{*} N^{p-1} / p N^{p-1}\right) \min \left\{c^{q}, c^{p}\right\}}{\sum_{k=1}^{N} \max _{|\xi| \leq c} F(k, \xi)}\right),
$$

the problem $\left(D_{\lambda}^{f}\right)$ admits at least two positive solutions.

Proof. Put $\Phi, \Psi$ as in (18). It is clear that $\inf _{X}(\Phi)=\Phi(0)=$ $\Psi(0)=0$. According to Lemma 3, we know that a nonzero critical point in $S$ of the functional $J_{\lambda}^{+}$is precisely a positive solution of problem $\left(D_{\lambda}^{f}\right)$. Next, we just need to prove condition (21) of Lemma 1.

We observe that $L_{\infty}>0$ from (40) and $\Lambda_{1}$ is nondegenerate. Fix $\lambda \in \Lambda_{1}$, Lemma 4 ensures that $J_{\lambda}$ satisfies
(PS)-condition for all $\lambda>2^{p} N+\widetilde{s}-2^{p-1} / q L_{\infty}$ and it is unbounded from below. We let $u \in \Phi^{-1}(-\infty, r]$, that is, $\left(\|u\|^{p} / p\right)+\left(\sum_{k=1}^{N} s(k)|u(k)|^{q} / q\right) \leq r$. Put

$$
r=\frac{1+s_{*} N^{p-1}}{p N^{p-1}} \min \left\{c^{q}, c^{p}\right\} .
$$

If $r=\left(1+s_{*} N^{p-1} / p N^{p-1}\right) c^{q}$, it means that $c \geq 1$. According to (8), we obtain

$$
|u(k)| \leq\|u\|_{\infty} \leq \max \left\{\left(\frac{p N^{p-1}}{1+s_{*} N^{p-1}}\right)^{1 / q} r^{1 / q},\left(\frac{p N^{p-1}}{1+s_{*} N^{p-1}}\right)^{1 / p} r^{1 / p}\right\}=\max \left\{c, c^{q / p}\right\}=c .
$$

If $r=\left(1+s_{*} N^{p-1} / p N^{p-1}\right) c^{p}$, we know $0<c<1$, then

$$
|u(k)| \leq\|u\|_{\infty} \leq \max \left\{\left(\frac{p N^{p-1}}{1+s_{*} N^{p-1}}\right)^{1 / q} r^{1 / q},\left(\frac{p N^{p-1}}{1+s_{*} N^{p-1}}\right)^{1 / p} r^{1 / p}\right\}=\max \left\{c^{p / q}, c\right\}=c
$$

To sum up, we know that $|u(k)| \leq c$ for all $k \in \mathbb{Z}(1, N)$. Furthermore, we have

$$
\Psi(u)=\sum_{k=1}^{N} F(k, u(k)) \leq \sum_{k=1}^{N} \max _{|\xi| \leq c} F(k, \xi),
$$

for all $u \in S$ with $\Phi(u) \leq r$. Hence,

$$
\frac{\sup _{u \in \Phi^{-1}(-\infty, r]} \Psi(u)}{r} \leq \frac{p N^{p-1}}{1+s_{*} N^{p-1}} \sum_{k=1}^{N} \max _{|\xi| \leq c} F(k, \xi) \max \left\{\frac{1}{c^{q}}, \frac{1}{c^{p}}\right\} .
$$


Now, let $\omega(k)=d$ for all $k \in \mathbb{Z}(1, N)$ and $\omega(0)=$ $\Delta \omega(N)=0$. Clearly, $\omega \in S$. It is easy to account that $\Phi(\omega)=$ $d^{p} p^{-1}+d^{q} q^{-1} \widetilde{\mathcal{s}}$, then

$$
\frac{\Psi(\omega)}{\Phi(\omega)}=\frac{\sum_{k=1}^{N} F(k, d)}{d^{p} p^{-1}+d^{q} q^{-1 \widetilde{\mathcal{S}}}} .
$$

Consequently, from (46), (47), and assumption (40), we can obtain

$$
\frac{\sup _{u \in \Phi^{-1}(-\infty, r]} \Psi(u)}{r}<\frac{\Psi(\omega)}{\Phi(\omega)} .
$$

Moreover, because $0<d<c$ and from (40), we obtain

$$
0<d^{p} p^{-1}+d^{q} q^{-1} \widetilde{\mathcal{s}}<\frac{1+s_{*} N^{p-1}}{p N^{p-1}} \min \left\{c^{q}, c^{p}\right\}
$$

that is mean that $0<\Phi(\omega)<r$.

Hence, the problem $\left(D_{\lambda}^{f}\right)$ admits at least two positive solutions by Lemma 1 and Lemma 3 for all $\lambda \in \Lambda_{1}$.

Remark 1. If $f(k, t)$ is a nonnegative function and there are two positive constants $c, d$ with $d<c$ such that

$$
\begin{array}{r}
\frac{p N^{p-1}}{1+s_{*} N^{p-1}} \max \left\{\frac{\sum_{k=1}^{N} F(k, c)}{c^{q}}, \frac{\sum_{k=1}^{N} F(k, c)}{c^{p}}\right\} \\
<\min \left\{\frac{\sum_{k=1}^{N} F(k, d)}{d^{p} p^{-1}+d^{q} q^{-1} \widetilde{s}}, \frac{q L_{\infty}}{2^{p} N+\widetilde{s}-2^{p-1}}\right\},
\end{array}
$$

then the result of Theorem 2 is also valid for each $\lambda \in \Lambda_{2}$ with

$$
\Lambda_{2}:=\left(\max \left\{\frac{d^{p} p^{-1}+d^{q} q^{-1} \widetilde{s}}{\sum_{k=1}^{N} F(k, d)}, \frac{2^{p} N+\widetilde{s}-2^{p-1}}{q L_{\infty}}\right\}, \frac{1+s_{*} N^{p-1}}{p N^{p-1}} \min \left\{\frac{c^{q}}{\sum_{k=1}^{N} F(k, c)}, \frac{c^{p}}{\sum_{k=1}^{N} F(k, c)}\right\}\right) .
$$

There are some consequences of Theorem 2 as follows.

Corollary 1. Let $g: \mathbb{R} \longrightarrow[0,+\infty)$ be a continuous function such that $f(k, t)=\alpha(k) g(t)$, where $\alpha(k)>0$ for all $k \in \mathbb{Z}(1, N)$. Put $A=\sum_{k=1}^{N} \alpha(k), G(t)=\int_{0}^{t} g(\xi) d \xi$ for all $t \in \mathbb{R}$ and $L_{\infty}^{*}:=\min _{k \in[1, N]} \alpha(k) \liminf _{t \rightarrow+\infty}\left(G(t) / t^{P}\right)>0$.

If there exists $c>d>0$ such that

$$
\begin{aligned}
& \frac{p N^{p-1}}{1+s_{*} N^{p-1}} A G(c) \max \left\{\frac{1}{c^{q}}, \frac{1}{c^{p}}\right\} \\
& \quad<\min \left\{\frac{A G(d)}{d^{p} p^{-1}+d^{q} q^{-1} \widetilde{\mathcal{s}}}, \frac{q L_{\infty}^{*}}{2^{p} N+\widetilde{s}-2^{p-1}}\right\},
\end{aligned}
$$

then the problem $D_{\lambda}^{f}$ has at least two positive solutions for each $\lambda \in \Lambda_{3}$ with

$$
\Lambda_{3}:=\left(\max \left\{\frac{d^{p} p^{-1}+d^{q} q^{-1} \widetilde{s}}{A G(d)}, \frac{2^{p} N+\widetilde{s}-2^{p-1}}{q L_{\infty}^{*}}\right\}, \frac{1+s_{*} N^{p-1}}{p N^{p-1}} \frac{\min \left\{c^{q}, c^{p}\right\}}{A G(c)}\right) .
$$

Proof. Consider the function $f: \mathbb{Z}(1, N) \times \mathbb{R} \longrightarrow \mathbb{R}$ is given as

$$
f(k, \xi)=\alpha(k) g(\xi), \quad \forall k \in \mathbb{Z}(1, N), \xi \in \mathbb{R},
$$

so that

$$
\sum_{k=1}^{N} \max _{|\xi| \leq c} F(k, \xi)=A G(c), \sum_{k=1}^{N} F(k, d)=A G(d) .
$$

Then, the conclusion can be obtained by Theorem 2 .

Corollary 2. Assume $f$ be a continuous function with $f(k, 0) \geq 0$ and

$$
\begin{gathered}
\limsup _{t \rightarrow 0^{+}} \frac{F(k, t)}{t^{p}}=+\infty, \\
\lim _{t \rightarrow+\infty} \frac{F(k, t)}{t^{p}}=+\infty,
\end{gathered}
$$

for all $k \in \mathbb{Z}(0, N)$. Put $\lambda^{*}=\left(1+s_{*} N^{p-1} / p N^{p-1}\right) \min \left\{s u p_{c>0}\right.$ $\left.\left(c^{q} / \sum_{k=1}^{N} \max _{|\xi| \leq c} F(k, \xi)\right), \sup _{c>0}\left(c^{p} / \sum_{k=1}^{N} \max _{|\xi| \leq c} F(k, \xi)\right)\right\}$.
Then, for each $\lambda \in\left(0, \lambda^{*}\right)$, the problem $\left(D_{\lambda}^{f}\right)$ admits at least two positive solutions.

Proof. We know that $L_{\infty}=+\infty$ from (57). Fix $\lambda \in\left(0, \lambda^{*}\right)$, and then there exists $c>0$ such that

$$
\begin{aligned}
\lambda< & \frac{1+s_{*} N^{p-1}}{p N^{p-1}} \\
& \cdot \min \left\{\sup _{c>0} \frac{c^{q}}{\sum_{k=1}^{N} \max _{|\xi| \leq c} F(k, \xi)}, \sup _{c>0} \frac{c^{p}}{\sum_{k=1}^{N} \max _{|\xi| \leq c} F(k, \xi)}\right\} .
\end{aligned}
$$

From (56), we can also obtain

$$
\underset{t \rightarrow 0^{+}}{\limsup } \frac{\sum_{k=1}^{N} F(k, t)}{t^{p}}=+\infty,
$$

and then there exists $d \in(0, c)$ such that $\left(\sum_{k=1}^{N} F(k, d) /\right.$ $\left.d^{p} p^{-1}+d^{q} q^{-1} \widetilde{s}\right)>(1 / \lambda)$. Therefore, Theorem 2 ensures the conclusion. 
Remark 2. If $f(k, t)$ is a nonnegative function for all $(k, t) \in \mathbb{Z}(1, N) \times[0,+\infty)$ As long as condition (56) holds for at least one $k \in \mathbb{Z}(1, N)$, then Corollary 2 ensures that the solutions are obtained for each

$$
\lambda \in\left(0, \frac{1+s_{*} N^{p-1}}{p N^{p-1}} \min \left\{\sup _{c>0} \frac{c^{q}}{\sum_{k=1}^{N} F(k, c)}, \sup _{c>0} \frac{c^{p}}{\sum_{k=1}^{N} F(k, c)}\right\}\right) .
$$

Remark 3. When $f(k, t)=f(t)$ for all $k \in \mathbb{Z}(1, N)$, Theorem 1 can be ensured by Corollary 2. Obviously, condition (4(a)) implies $f(0) \geq 0$. Specially, if $f$ is nonnegative, we only need condition (4(a)) to get the corresponding solution for each

$$
\lambda \in\left(0, \frac{1+s_{*} N^{p-1}}{p N^{p}} \min \left\{\sup _{c>0} \frac{c^{q}}{F(c)}, \sup _{c>0} \frac{c^{p}}{F(c)}\right\}\right) .
$$

Example 1. Let $p=4, q=2, N=3, s(k)=12$, and $f(k, t)$ $=e^{t}$.

Put

$$
x(c)=\frac{c^{2}}{e^{c}-1} .
$$

Then,

$$
x^{\prime}(c)=\frac{c\left((2-c) e^{c}-2\right)}{\left(e^{c}-1\right)^{2}} .
$$

Let $z(c)=(2-c) e^{c}-2$, then $z^{\prime}(c)=(1-c) e^{c}$. So, $z(c)$ is increasing in $c \in(0,1)$ and decreasing in $c \in(1,+\infty)$. Since $z(0)=0$ and $z(+\infty)=-\infty$, there exists an unique $c_{1} \in(1,+\infty)$ such that $z\left(c_{1}\right)=0$. Thus, $x(c)$ in increasing in $c \in\left(0, c_{1}\right)$ and decreasing in $c \in\left(c_{1},+\infty\right)$. This means that $\sup _{c>0} x(c)=x\left(c_{1}\right)$. In fact, $c_{1} \approx 1.5936$.

Similarly, put $y(c)=c^{4} / e^{c}-1$, we can show that there exists a unique $c_{2} \in(3,+\infty)$ such that $\sup _{c>0} y(c)=y\left(c_{2}\right)$. In fact, $c_{2} \approx 3.9207$.

Since

$$
y\left(c_{2}\right)=\frac{c_{2}^{4}}{e^{c_{2}}-1}>\frac{c_{1}^{4}}{e^{c_{1}}-1}=c_{1}^{2} x\left(c_{1}\right)>x\left(c_{1}\right),
$$

then

$$
\begin{gathered}
\frac{1+s_{*} N^{p-1}}{p N^{p}} \min \left\{\sup _{c>0} x(c), \sup _{c>0} y(c)\right\} \\
=\frac{1+s_{*} N^{p-1}}{p N^{p}} x\left(c_{1}\right) \approx 0.6496 .
\end{gathered}
$$

Therefore, for each $\lambda \in(0,0.6496)$, the problem

$$
\left\{\begin{array}{l}
-\Delta\left(\phi_{4}(\Delta u(k-1))\right)+12 \phi_{2}(u(k))=\lambda e^{u(k)}, \quad k=1,2,3, \\
u(0)=\Delta u(3)=0,
\end{array}\right.
$$

admits at least two positive solutions.
Example 2. Let $N=3, p=3$, and $q=2$ and $f$ be a function as follows:

$$
f(k, t)= \begin{cases}0, & \text { if } t<0, \\ \sqrt[3]{k t}, & \text { if } 0 \leq t \leq 1, \\ \sqrt[3]{k t}+225 t^{2}-225, & \text { if } t>1\end{cases}
$$

From Remark 1, we can choose $c=1$ and $d=0.02$. Easy calculation shows that

$$
\begin{aligned}
\frac{p N^{p-1}}{1+s_{*} N^{p-1}} \sum_{k=1}^{N} F(k, c) \max \left\{\frac{1}{c^{q}}, \frac{1}{c^{p}}\right\} & =\frac{p N^{p-1}}{1+s_{*} N^{p-1}} \sum_{k=1}^{3} \int_{0}^{1} \sqrt[3]{k t} \mathrm{~d} t \\
& \approx 1.3693, \\
\frac{\sum_{k=1}^{N} F(k, d)}{d^{p} p^{-1}+d^{q} q^{-1} \widetilde{\mathcal{S}}} & =\frac{\sum_{k=1}^{3} \int_{0}^{0.02} \sqrt[3]{k t} \mathrm{~d} t}{d^{p} p^{-1}+d^{q} q^{-1} \widetilde{\mathcal{S}}} \approx 3.1386, \\
\frac{q L_{\infty}}{2^{p} N+\widetilde{s}-2^{p-1}} & =\frac{1}{66} \lim _{t \rightarrow+\infty} \frac{\sqrt[3]{k t}+225 t^{2}-225}{t^{2}} \\
& \approx 3.4091,
\end{aligned}
$$

which satisfy condition (50). Thus, for each $\lambda \epsilon$ $(0.3186,0.7303)$, the problem

$$
\left\{\begin{array}{l}
-\Delta\left(\phi_{3}(\Delta u(k-1))\right)+8 \phi_{2}(u(k))=\lambda f(k, u(k)), \quad k=1,2,3, \\
u(0)=\Delta u(3)=0,
\end{array}\right.
$$

admits at least two positive solutions.

\section{Data Availability}

No data were used to support the study.

\section{Conflicts of Interest}

The authors declare that there are no conflicts of interest regarding the publication of this paper.

\section{Authors' Contributions}

All authors contributed equally to the writing of this paper. All authors read and approved the final manuscript.

\section{Acknowledgments}

This work was supported by the National Natural Science Foundation of China (Grant no. 11971126) and Program for Changjiang Scholars and Innovative Research Team in University (Grant no. IRT_16R16).

\section{References}

[1] R. P. Agarwal, Difference Equations and Inequalities. Theory, Methods, and Applications, Marcel Dekker, Inc., New York, NY, USA, 2000.

[2] A. Kristály, M. Mihăilescu, V. Rădulescu, and S. Tersian, "Spectral estimates for a nonhomogeneous difference 
problem," Communications in Contemporary Mathematics, vol. 12, no. 6, pp. 1015-1029, 2010.

[3] L. M. Li and Z. Zhou, "Infinitely many positive solutions for a coupled discrete boundary value problem," Discrete Dynamics in Nature and Society, vol. 2019, Article ID 8052497, 7 pages, 2019.

[4] J. X. Ling and Z. Zhou, "Positive solutions of the discrete Dirichlet problem involving the mean curvature operator," Open Mathematics, vol. 17, no. 1, pp. 1055-1064, 2019.

[5] Q. Q. Zhang, "Homoclinic orbits for discrete Hamiltonian systems with local super-quadratic conditions," Communications on Pure \& Applied Analysis, vol. 18, no. 1, pp. 425-434, 2019.

[6] Z. Zhou and J. X. Ling, "Infinitely many positive solutions for a discrete two point nonlinear boundary value problem with $\phi_{c}$-Laplacian," Applied Mathematics Letters, vol. 91, pp. 2834, 2019.

[7] G. Bonanno and P. Candito, "Infinitely many solutions for a class of discrete non-linear boundary value problems," Applicable Analysis, vol. 88, no. 4, pp. 605-616, 2009.

[8] G. H. Lin and Z. Zhou, "Homoclinic solutions of discrete $\varphi$-Laplacian equations with mixed nonlinearities," Communications on Pure \& Applied Analysis, vol. 17, no. 5, pp. 1723-1747, 2018.

[9] J. S. Yu and B. Zheng, "Modeling Wolbachia infection in mosquito population via discrete dynamical model," Journal of Difference Equations and Applications, vol. 25, no. 11, pp. 1549-1567, 2019.

[10] P. Mei, Z. Zhou, and G. H. Lin, "Periodic and subharmonic solutions for a $2 n$ th-order $\phi_{c}$-Laplacian difference equation containing both advances and retardations," Discrete Cont Dyn-S, vol. 12, pp. 2085-2095, 2019.

[11] M. K. Moghadam, L. Li, and S. Tersian, "Existence of three solutions for a discrete anisotropic boundary value problem," Bulletin of the Iranian Mathematical Society, vol. 44, no. 4, pp. 1091-1107, 2018.

[12] L. Erbe, B. G. Jia, and Q. Q. Zhang, "Homoclinic solutions of discrete nonlinear systems via variational method," Journal of Applied Analysis and Computation, vol. 9, pp. 271-294, 2019.

[13] S. Heidarkhani and M. Imbesi, "Multiple solutions for partial discrete Dirichlet problems depending on a real parameter," Journal of Difference Equations and Applications, vol. 21, no. 2, pp. 96-110, 2015.

[14] G. H. Lin, Z. Zhou, and J. S. Yu, "Ground state solutions of discrete asymptotically linear Schrödinge equations with bounded and non-periodic potentials," Journal of Dynamics and Differential Equations, 2019.

[15] M. Mihăilescu, V. Rădulescu, and S. Tersian, "Homoclinic solutions of difference equations with variable exponents," Topological Methods in Nonlinear Analysis, vol. 38, pp. 277289, 2011.

[16] J. Kuang and Z. Guo, "Heteroclinic solutions for a class of $p$ Laplacian difference equations with a parameter," Applied Mathematics Letters, vol. 100, Article ID 106034, 2020.

[17] C. Bereanu and J. Mawhin, "Boundary value problems for second-order nonlinear difference equations with discrete $\varphi$-Laplacian and singular $\varphi$," Journal of Difference Equations and Applications, vol. 14, no. 10-11, pp. 1099-1118, 2008.

[18] W. G. Kelly and A. C. Peterson, Difference Equations: An Introduction with Applications, Academic Press, San Diego, CA, USA, 1991.

[19] G. Bonanno, P. Jebelean, and C. Şerban, "Superlinear discrete problems," Applied Mathematics Letters, vol. 52, pp. 162-168, 2016.
[20] J. Henderson and H. B. Thompson, "Existence of multiple solutions for second-order discrete boundary value problems," Computers \& Mathematics with Applications, vol. 43, no. 10-11, pp. 1239-1248, 2002.

[21] Y. H. Long and J. L. Chen, "Existence of multiple solutions to second-order discrete Neumann boundary problems," Applied Mathematics Letters, vol. 83, pp. 7-14, 2018.

[22] B. Ricceri, "A general variational principle and some of its applications," Journal of Computational and Applied Mathematics, vol. 133, no. 1-2, pp. 401-410, 2000.

[23] Z. Zhou and D. F. Ma, "Multiplicity results of breathers for the discrete nonlinear Schrödinger equations with unbounded potentials," Science China Mathematics, vol. 58, no. 4, pp. 781-790, 2015.

[24] R. P. Agarwal, K. Perera, and D. O'Regan, "Multiple positive solutions of singular discrete $p$-Laplacian problems via variational methods," Advances in Difference Equations, vol. 2015, no. 2, Article ID 690272, 2005.

[25] A. Nastasi, C. Vetro, and F. Vetro, "Positive solutions of discrete boundary value problems with the $(p, q)$-Laplacian operator," Electronic Journal of Differential Equations, vol. 225, pp. 1-12, 2017.

[26] Z. Zhou and M. T. Su, "Boundary value problems for $2 n$ thorder $\phi_{c}$-Laplacian difference equations containing both advance and retardation," Applied Mathematics Letters, vol. 41, pp. 7-11, 2015.

[27] Z. Zhou, J. S. Yu, and Y. M. Chen, "Homoclinic solutions in periodic diffrence equations with saturable nonlinearity," Science China Mathematics, vol. 54, no. 1, pp. 83-93, 2011.

[28] G. D’Aguì, J. Mawhin, and A. Sciammetta, "Positive solutions for a discrete two point nonlinear boundary value problem with p-Laplacian," Journal of Mathematical Analysis and Applications, vol. 447, no. 1, pp. 383-397, 2017.

[29] G. D’Aguì, A. Sciammetta, and E. Tornatore, “Two non-zero solutions for Sturm-Liouville equations with mixed boundary condition," Nonlinear Anal Real World Appl, vol. 47, pp. 324-331, 2019.

[30] G. Bonanno and G. D’Aguì, "Two non-zero solutions for elliptic Dirichlet problems," Zeitschrift für Analysis und ihre Anwendungen, vol. 35, no. 4, pp. 449-464, 2016.

[31] G. Bonanno, "A critical point theorem via the Ekeland variational principle," Nonlinear Analysis: Theory, Methods \& Applications, vol. 75, no. 5, pp. 2992-3007, 2012.

[32] A. Ambrosetti and P. H. Rabinowitz, "Dual variational methods in critical point theory and applications," Journal of Functional Analysis, vol. 14, no. 4, pp. 349-381, 1973.

[33] G. Bonanno, P. Candito, and G. D'Aguì, "Variational methods on finite dimensional Banach space and discrete problems," Advanced Nonlinear Studies, vol. 14, no. 4, pp. 915-939, 2014. 